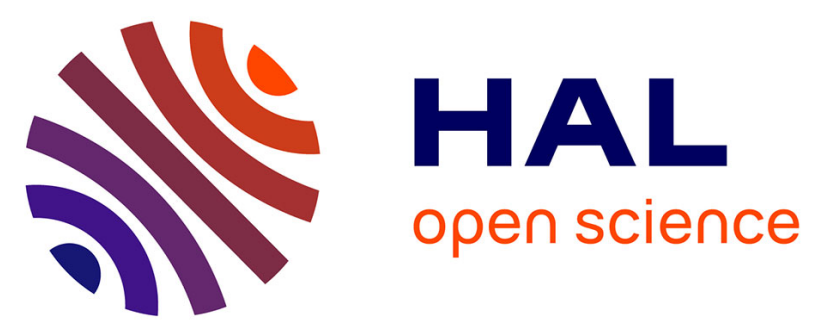

\title{
Emission and detection of terahertz radiation using two-dimensional plasmons in semiconductor nanoheterostructures for nondestructive evaluations
}

Taiichi Otsuji, Takayuki Watanabe, Stephane Albon Boubanga Tombet, Akira Satou, Victor Ryzhii, Vyacheslav Popov, Wojciech Knap

\section{To cite this version:}

Taiichi Otsuji, Takayuki Watanabe, Stephane Albon Boubanga Tombet, Akira Satou, Victor Ryzhii, et al.. Emission and detection of terahertz radiation using two-dimensional plasmons in semiconductor nanoheterostructures for nondestructive evaluations. Optical Engineering, 2014, 53 (3), pp.031206. 10.1117/1.OE.53.3.031206 . hal-00954505

\section{HAL Id: hal-00954505 https://hal.science/hal-00954505}

Submitted on 8 Jun 2021

HAL is a multi-disciplinary open access archive for the deposit and dissemination of scientific research documents, whether they are published or not. The documents may come from teaching and research institutions in France or abroad, or from public or private research centers.
L'archive ouverte pluridisciplinaire HAL, est destinée au dépôt et à la diffusion de documents scientifiques de niveau recherche, publiés ou non, émanant des établissements d'enseignement et de recherche français ou étrangers, des laboratoires publics ou privés. 


\section{Optical Engineering}

\section{Emission and detection of terahertz radiation using two-dimensional plasmons in semiconductor nanoheterostructures for nondestructive evaluations}

Taiichi Otsuji

Takayuki Watanabe

Stephane Albon Boubanga Tombet

Akira Satou

Victor Ryzhii

Vyacheslav Popov

Wojciech Knap 


\section{Emission and detection of terahertz radiation using two-dimensional plasmons in semiconductor nanoheterostructures for nondestructive evaluations}

\author{
Taiichi Otsuji \\ Takayuki Watanabe \\ Stephane Albon Boubanga Tombet \\ Akira Satou \\ Victor Ryzhii \\ Tohoku University \\ Research Institute of Electrical Communication \\ 9808577 Sendai, Japan \\ E-mail: otsuji@ riec.tohoku.ac.jp \\ Vyacheslav Popov \\ Russian Academy of Science \\ Kotelnikov Institute of Radio Engineering and \\ Electronics (Saratov Branch) \\ 410019 Saratov, Russia \\ Wojciech Knap \\ University of Montpellier-CNRS \\ LC2 Laboratories \\ UMR 5221 \\ 34095 Montpellier, France
}

\begin{abstract}
The recent advances in emission and detection of terahertz radiation using two-dimensional (2-D) plasmons in semiconductor nanoheterostructures for nondestructive evaluations are reviewed. The 2-D plasmon resonance is introduced as the operation principle for broadband emission and detection of terahertz radiation. The device structure is based on a high-electron-mobility transistor and incorporates the authors' original asymmetrically interdigitated dual-grating gates. Excellent $\mathrm{THz}$ emission and detection performances are experimentally demonstrated by using InAIAs/InGaAs/InP and/or InGaP/InGaAs/GaAs heterostructure material systems. Their applications to nondestructive material evaluation based on $\mathrm{THz}$ imaging are also presented. () The Authors. Published by SPIE under a Creative Commons Attribution 3.0 Unported License. Distribution or reproduction of this work in whole or in part requires full attribution of the original publication, including its DOI. [DOI: 10.1117/1.OE.53.3.031206]
\end{abstract}

Subject terms: terahertz; emission; detection; plasmons; nondestructive.

Paper 130761SSP received May 23, 2013; revised manuscript received Aug. 31, 2013; accepted for publication Oct. 28, 2013; published online Nov. 27, 2013.

\section{Introduction}

In the research of modern terahertz (THz) electronics, development of compact, tunable, and coherent sources operating at $\mathrm{THz}$ frequencies is one of the hottest issues. ${ }^{1}$ Two-dimensional (2-D) plasmons in submicron transistors have attracted much attention due to their nature of promoting emission and detection of electromagnetic radiation in the $\mathrm{THz}$ range. The channel of a transistor can act as a resonator for plasma waves, the charge-density waves of collectively excited 2-D electrons. The plasma frequency depends on the resonator dimensions and the density of 2-D electrons. It can reach the sub-THz or even $\mathrm{THz}$ range for gate lengths of a micron and submicron (nanometer) size. Therefore, different devices/structures of micron and submicron sizes supporting low-dimensional plasmons were intensively studied as possible candidates for solid-state far-infrared (FIR)/THz sources $^{2-18}$ and detectors. ${ }^{19-30}$ Mechanisms of plasma wave excitation/emission can be divided (by convention) into two types: (1) incoherent and (2) coherent. The first is related to thermal excitation of broadband nonresonant plasmons by hot electrons. ${ }^{2-7}$ The second is related to the plasma wave instability mechanisms like Dyakonov-Shur (DS) Doppler-shift model ${ }^{8}$ and/or Ryzhii-Satou-Shur (RSS) transit-time model, ${ }^{17,18}$ where coherent plasmons can be excited either by hot electrons or by optical phonon emission under near ballistic electron motion. ${ }^{31}$ On the other hand, hydrodynamic nonlinearities of 2-D plasmons in highelectron-mobility transistors (HEMTs) are promising for fast and sensitive rectification/detection of $\mathrm{THz}$ radiation. ${ }^{19}$ When an incoming $\mathrm{THz}$ radiation excites the plasma waves, the local carrier density as well as the local carrier drift velocity is modulated by the radiation frequency. This results in generation of the quadratic current term proportional to the product of the modulated components of the local carrier densities and velocities. The time average of this component is nonzero, leading to rectification. With an asymmetric boundary condition, a rectified component gives rise to a photovoltaic effect. Recently, plasma wave properties were successfully used for the resonant and nonresonant (broadband) sub-THz and THz detection. ${ }^{20-30}$ They can be applied to real-time $\mathrm{THz}$ imaging/spectroscopic analysis as well as future $\mathrm{THz}$ wireless communications. ${ }^{1}$

We have first proposed a 2-D plasmon-resonant microchip emitter featured with an interdigitated dual-grating gates (DGGs) structure. ${ }^{32-35}$ The original structure uses symmetrical DGGs in which interfinger spaces are all identical, providing room-temperature 0.5 - to $6.5-\mathrm{THz}$ emission with 1-microwat-order radiation power ${ }^{33,34}$ and rather low detection responsivity of the order of 10 's V/W. ${ }^{35}$ Recently, authors have proposed an asymmetric DGG (A-DGG) structure and demonstrated coherent monochromatic $\mathrm{THz}$ emission and ultrahigh-sensitive $\mathrm{THz}$ detection with $2.2 \mathrm{kV} / \mathrm{W}$ at $1 \mathrm{THz}$ radiation. ${ }^{36-39}$ This paper reviews recent advances in emission and detection of $\mathrm{THz}$ radiation using 2-D plasmons in semiconductor nanoheterostructures for nondestructive evaluations. The 2-D plasmon resonance is first presented as the operation principle to demonstrate its potential of broadband intense emission and sensitive detection of $\mathrm{THz}$ radiation. Second, the device structure that can provide practical emission and detection performances are addressed, which is based on a HEMT and incorporates the authors' original asymmetrically interdigitated DGGs. Numerical analysis 
reveals that in comparison with conventional symmetric DGG (S-DGG) structure, the A-DGG can substantially improve the detection sensitivity as well as the instability (emissivity) by three to four orders of magnitude. Third, excellent $\mathrm{THz}$ emission and detection performances including coherent, monochromatic emission beyond $1-\mathrm{THz}$ range $^{39}$ and an extremely high detection responsivity of $6.4 \mathrm{kV} / \mathrm{W}$ at $1 \mathrm{THz}$ at $300 \mathrm{~K}$ (Ref. 40) are experimentally demonstrated by using InAlAs/InGaAs/InP heterostructure material systems. Finally, their applications to nondestructive material evaluation based on $\mathrm{THz}$ imaging are demonstrated.

\section{THz Emission Using 2-D Plasmons in HEMTs}

\subsection{Theory}

In a simple case, the 2-D plasma wave motions can be described by the hydrodynamic Euler equation and the continuity equation. ${ }^{8}$

$m\left(\frac{\partial \mathbf{u}}{\partial t}+\mathbf{u} \frac{\partial \mathbf{u}}{\partial \mathbf{r}}\right)=-e \frac{\partial V}{\partial \mathbf{r}}-\frac{\mathbf{u}}{m \tau}$,

$\frac{\partial n}{\partial t}+\frac{\partial}{\partial \mathbf{r}}(n \mathbf{u})=0$

where $m$ is the electron effective mass, $\mathbf{r}$ is an arbitrary inplane vector, $\mathbf{u}(\mathbf{r}, t)$ is the in-plane electron spatiotemporal local velocity, $\tau$ is the electron momentum relaxation time, $V(\mathbf{r}, t)$ is the local potential at $\mathbf{r}$, and $n(\mathbf{r}, t)$ is the spatiotemporal local density of electrons. The first term of the right-hand in Eq. (1) is the Coulomb force and the second term is the Drude friction. 2-D electron channels in HEMTs consisting of gated and ungated regions as shown in Fig. 1. The ungated 2-D plasmon receives the in-plane longitudinal Coulomb force so that it holds a square-root dispersion relation, which is identical to that for general surface plasmons. The gated 2-D plasmon receives transverse Coulomb force via gate capacitor, which is far stronger than the in-plane
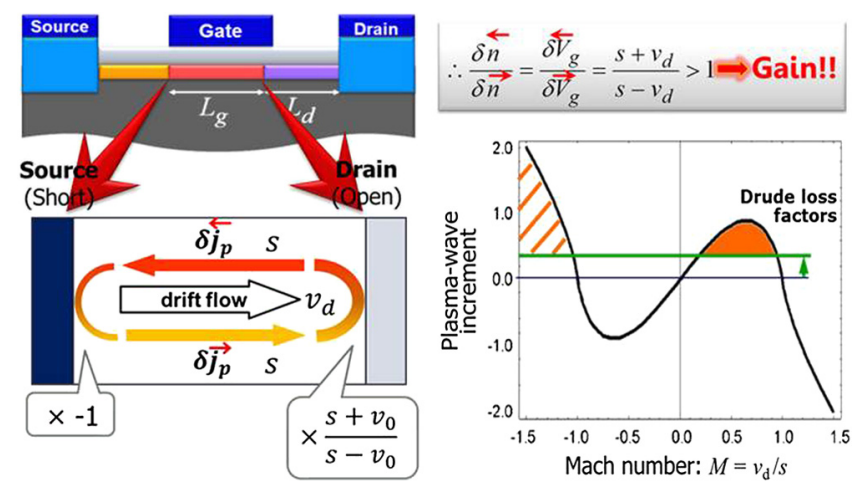

Fig. 1 DS type plasma-wave instability in a two-dimensional (2-D) electron channel under source-terminated and drain-opened boundaries with dc drift velocity $v_{d} \cdot{ }^{8}$ The reciprocal Doppler-shifting plasma waves reflecting at an asymmetric drain-opened boundary promote the increments of their intensity leading to self-oscillation of instability. The plasma-wave increment is a dimensionless parameter, in which the imaginary part of angular frequency is normalized to the fundamental resonant frequency. The positive values of the increment give rise to instability in an idealistic lossless case. In reality, with finite $\tau$ value, the Drude loss factor should consider to obtain an overall gain, which is shown as a threshold level. force due to the geometrical situation so that it holds a linear dispersion. In a simple case of gradual-channel approximation with infinite channel width (perpendicular to the sourcedrain direction), the 2-D plasma wave dynamics can be reduced to one-dimensional systems. ${ }^{8}$ The plasma wave phase velocity $s$ is given by $s=\sqrt{e V_{0} / m}$, where $V_{0}$ is the gate swing voltage. ${ }^{19}$ Assuming $V_{0}=O[1 \mathrm{~V}]$ and $m=$ $O\left[0.1 m_{0}\right]\left(m_{0}\right.$ is the electron rest mass in vacuum) for InPbased heterostructure HEMTs, $s$ becomes $O\left[1 \times 10^{6} \mathrm{~m} / \mathrm{s}\right]$, which is at least two orders of magnitude higher than the electron drift velocity of any compound semiconductors with superior transport properties. Thus, when we consider a sub-micrometer gate-length HEMT, the fundamental mode of gated 2-D plasmons stays at a frequency in the THz range. This is the main advantage for use in plasmon-resonant modes that can operate in the frequencies far beyond the transit frequency limit of transistors.

When a single-gate HEMT is situated in source-terminated and drain-opened configuration with dc potential at the drain terminal with respect to the source terminal, the drain end of the channel becomes depleted so that the drainside impedance is mainly given by the depletion capacitance and takes a high value at high frequencies. In such a case, a Doppler-shift effect occurs on the plasma wave propagation/ reflection at the drain boundary, promoting the DS instability. ${ }^{8}$ Consider the case in which a plasma wave is excited in an HEMT with a constant de drain bias causing a background constant dc electron drift flow with velocity $v_{d}$ and the gate length $L$ is shorter than the coherent length of electrons. The plasma wave-originated local displacement current $\delta j_{p}$ is given by the product of the perturbation of the local electron charge density $e \delta n$ and the plasma wave velocity. The forward (backward) component $\delta \rightarrow j_{p}\left(\delta \leftarrow j_{p}\right)$ traveling to (from) the drain boundary is given by $\delta \rightarrow j_{p}=$ $e \delta \rightarrow n \cdot\left(s+v_{d}\right), \delta \leftarrow j_{p}=e \delta \leftarrow n \cdot\left(s-v_{d}\right)$. Since the opendrain boundary conserves the current before and after the reflection, $\left|\delta \rightarrow j_{p}\right|=\left|\delta \leftarrow j_{p}\right|, \delta \leftarrow n=\frac{s+v_{d}}{s-v_{d}} \cdot \delta \leftarrow n>\delta \leftarrow n$. This increment of the electron charge density $\delta n=(\delta \leftarrow n-$ $\delta \rightarrow n)$ directly reflects the increment of the gate potential $\delta V_{g}$ via gate capacitor $C: \delta V_{g}=e \delta n \cdot C$. Since the sourceterminated boundary gives a lossless reflection (reflection coefficient is -1 ), the gate potential becomes infinite after infinitesimal repetitions of plasma wave reflections, leading to the DS instability. When the plasma wave is excited by the incoming $\mathrm{THz}$ radiation with angular frequency $\omega$, $e^{-i \omega t}$, the effect of the instability is characterized by the imaginary part of $\omega, \omega^{\prime \prime}$, as

$\omega^{\prime \prime}=\frac{s^{2}-v_{d}^{2}}{L s} \ln \left|\frac{s+v_{d}}{s-v_{d}}\right|$.

When $\omega^{\prime \prime}>0$, the system becomes unstable, giving rise to the condition of the DS instability. The plasma wave increment in units of $s / 2 L$, a dimensionless plasma wave increment $2 \omega^{\prime \prime} L / s$ is plotted as a function of the Mach number $s / v_{d}{ }^{8}$ In reality, with finite $\tau$ value, the Drude loss factor should be considered to obtain an overall gain, which is shown as a threshold level in Fig. 1. So far DS-instability-driven voltage-tunable millimeter-wave to THzwave emission has been observed at low and room temperatures from GaAs-, InP-, and GaN-based HEMTs. ${ }^{9-12}$ 
When the channel pinch-off is insufficient and the drain terminal is not open but yet conductive at $\mathrm{THz}$ frequencies, the plasma waves are effectively absorbed in the high-field gate-drain region. Hence their reflection is insufficient to promote necessary positive feedback for the occurrence of the DS instability. At large drain-source voltages, the $\mathrm{THz}$ conductivity of this region would be rather high due to the relatively high value of the electron drift velocity $v_{g d}$ in the high-field gate-drain region. In this case, $v_{g d}$ becomes much higher than that in the intrinsic channel region $v_{d}$. Note that the electrons propagating in the high-field gatedrain region induce the ac current in the gated channel and the drain contact. One can find that the electron ac concentration as a function of the coordinate varies as $n_{\omega}(x)=$ $\left.n_{\omega}\right|_{x=L_{g}} e^{-i \omega(x-L g) / v_{g d} .}{ }^{17,18}$ As a result, the ac current induced in the gated channel is presented. Its frequency dependence is directly reflected by the electron transit time $\tau_{d d}$ at the gate-drain region $\tau_{g d}=L_{d} / v_{g d}$, where $L_{d}$ is the length of the gate-drain region, which may contribute to promote the plasma wave instability. ${ }^{17}$ In this case, the instability condition is given by the following inequality using the imaginary part of the plasma wave current:

$\operatorname{Im}(\omega)=\omega^{\prime \prime} \simeq-\frac{\nu}{2}+\gamma_{\mathrm{DS}}+\gamma_{\mathrm{RSS}}>0$

$\gamma_{\mathrm{DS}} \simeq \frac{v_{d}}{L g}$

$\gamma_{\mathrm{RSS}} \simeq-\frac{v_{g d}}{L_{g}} r \cos \left(\omega_{n} \tau_{g d} / 2\right) J_{0}\left(\omega_{n} \tau_{g d} / 2\right)$,

where $\nu$ is the electron collision frequency, $r$ is a phenomenological parameter $(r \leq 1), J_{0}$ is the zeroth Bessel function, and $n=1,2,3, \ldots$ is the plasmon mode index. ${ }^{17}$ Here, $\gamma_{\text {DS }}$ and $\gamma_{\text {RSS }}$ are the DS-instability index and the transit-time-driven RSS-instability index, respectively. $\gamma_{\text {RSS }}$ can take both positive and negative values depending on $\omega_{n} \tau_{g d}$. The contributions to the plasma wave instability growth rate of the DS and RSS mechanism $\left(\gamma_{\mathrm{DS}} / 2 \pi\right.$ and $\left.\gamma_{\mathrm{RSS}} / 2 \pi\right)$ in an HEMT with typical geometric and material parameters are plotted in Fig. 2 as functions of the fundamental plasmon mode frequency. ${ }^{17}$ Due to the nature of transit-time-driven mechanism, the RSS instability is sensitive to the plasmon mode frequency.

The 2-D plasmon itself is a nonradiative mode so that a metal-wired grating coupler structure is frequently utilized to yield THz electromagnetic-wave emission. ${ }^{2-7}$ If the single gate is replaced with a grating-finger-type gate, a plural number of plasmonic cavities (whose electron density is modulated by the bias voltage of the grating-finger gate) are electrostatically coupled (via interfinger region with less electron density) in a spatially distributed configuration. In this case, a constant dc channel current, which is generated by the applied dc drain bias, gives rise to periodic electron velocity modulation over the channel. This may also promote the RSS instability. ${ }^{18}$

In particular, the DGG structure provides an improved confinement of the 2-D electrons into the plasmon cavities independent of the tuned gate biases, resulting in more intense resonant plasma excitation with higher quality factors [see Fig. 3(a)]. ${ }^{32-34}$ The DGGs can alternately modulate the 2-D electron densities to periodically distribute the plasmonic cavities ( 100 nm width in microns distance) along the channel by applying a large fraction of gate biases for subgratings G1 and G2. ${ }^{32-34}$ Under pertinent drain-source $\mathrm{dc}$ bias conditions, dc electron drift flows may promote the plasmon instability, resulting in self-oscillation with characteristic frequencies in the $\mathrm{THz}$ regime. Figure 3(b) depicts the potential distribution and equivalent circuit model for the RSS instability under a constant dc-drain bias condition. The 2-D electron channel consists of a periodic series of highly confined 2-D plasmon cavity sections underneath the gate finger $\mathrm{G} 1$ and a depleted section underneath the gate finger $\mathrm{G} 2$.

According to the aforementioned RSS instability mechanism, the depleted barrier section works for the negative conductance gain section, whereas the highly confined section works for the plasmonic resonator modeled by an $L-C-R$ circuitry as shown in Fig. 3(b) ${ }^{38}$ The model accounts for the features of the electron transport across the device structure with the 2D-electron stripes (2DESs) that are formed underneath one grating gate fingers separated by the depleted barrier regions and the possibility of the plasma oscillations in the 2DESs electrically coupled via the barrier regions. We demonstrated that, due to the negative dynamic conductance of the barrier regions associated with the electron transit-time effects, the plasma instability leading to the self-excitation of
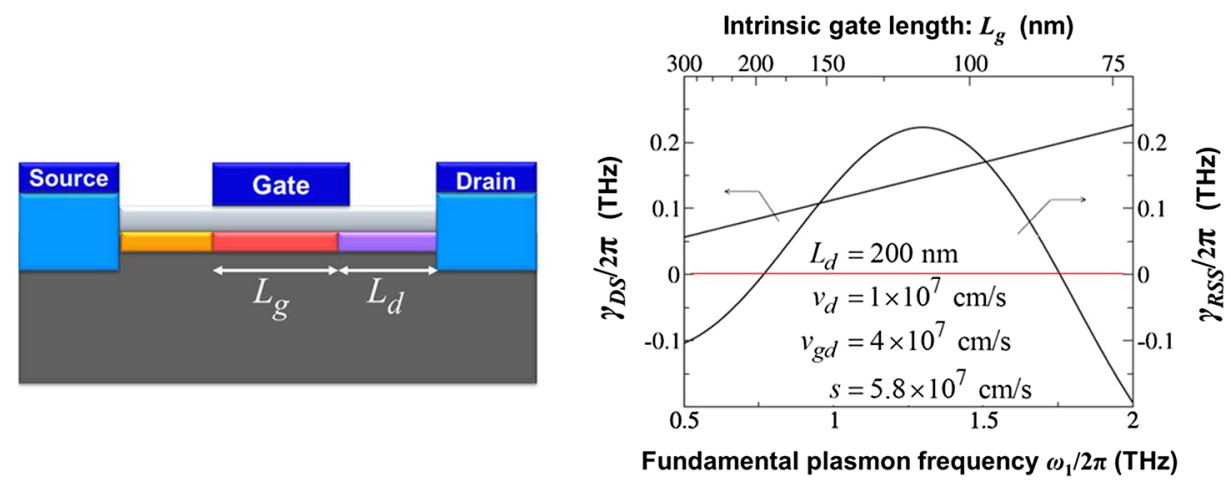

Fig. 2 Contributions to the plasma-wave instability growth rate of the DS and RSS mechanism $\left(\gamma_{\mathrm{DS}} / 2 \pi\right.$ and $\left.\gamma_{\mathrm{RSS}} / 2 \pi\right)$ in a high-electron-mobility transistor (HEMT) with typical geometric and material parameters as functions of the fundamental plasmon mode frequency. ${ }^{17}$ Due to the nature of transit-time-driven mechanism, the RSS instability is sensitive to the plasmon mode frequency. 


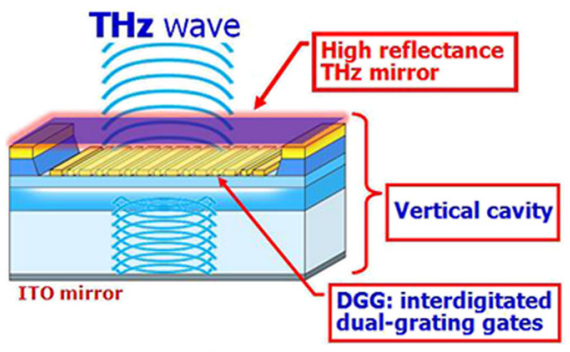

(a)

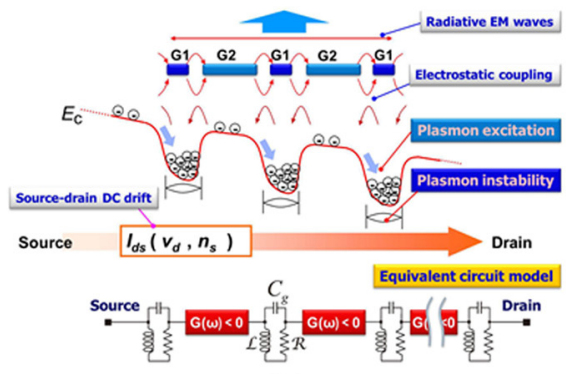

(b)

Fig. 3 Dual-grating gate (DGG)-HEMT-type terahertz (THz) emitter with a vertical cavity. (a) Structure. (b) Band diagram and equivalent circuit.

the plasma oscillations (and the emission of $\mathrm{THz}$ radiation) becomes feasible when the dc current exceeds a certain threshold value. ${ }^{33}$

\subsection{Experiments}

We have proposed our original DGG-HEMT-type 2-D plasmon-resonant microchip emitter as a new $\mathrm{THz}$ light source. $^{32-34,39}$ The original structure is a S-DGG in which interfinger spaces are all identical. The device was fabricated using InGaP/InGaAs/GaAs and/or InAl/InGaAs/InP material systems. ${ }^{33,34}$ So far a broadband $\mathrm{THz}$ emission ranging from 1 to $\sim 6 \mathrm{THz}$ with a maximum output power of $\sim 1 \mu \mathrm{W}$ at $300 \mathrm{~K}$ has been obtained reflecting multimode of coherent/incoherent plasmons, ${ }^{41}$ for which oblique modes, ${ }^{42}$ gated and ungated plasmon modes, ${ }^{43}$ hot plasmons, ${ }^{41}$ and chirped plasmon modes $^{33}$ are the major causes. The DGG-HEMT THz emitter can work for THz spectroscopic and imaging applications as an incoherent broadband $\mathrm{THz}$ microchip source, demonstrating fine identification of water vapor absorptions as well as finger prints of sugar groups. ${ }^{34}$

In order to realize coherent monochromatic $\mathrm{THz}$ emission, we introduced our original A-DGG structure [Figs. 4(a) and $4(\mathrm{~b})] .{ }^{36}$ In the A-DGG structure, the DGG is implemented with asymmetric interfinger spaces. $\mathrm{THz}$ electric field distribution and resultant photoresponse were numerically simulated using a self-consistent electromagnetic approach combined with the perturbation theory for the hydrodynamic equations for 2-D plasmons in HEMTs under periodic electron density modulation conditions [Figs. 4(c) and 4(d)]. ${ }^{36,39}$ Figure 4(c) shows giant enhancement of the responsiveness (by four orders of magnitude) in an A-DGG HEMT under drain-unbiased conditions with respect to that for a S-DGG HEMT for a de drain bias current density of $0.1 \mathrm{~A} / \mathrm{m}$. When the A-DGG HEMT is dc-drain biased, the asymmetry of the plasmonic cavity is enhanced enormously, resulting in further enhancement of the responsiveness by orders of magnitude. It is reasonable to suggest that a similar enhancement should also be exhibited for the plasmon instability in the A-DGG HEMT. A chirped DGG structure was also introduced so as to uniform the plasmon frequencies over the entire DGG area under a specific drainsource bias $V_{d s}$ condition. These two features are combined to serve an asymmetric chirped DGG (AC-DGG) structure. Moreover, a high-Q vertical photonic cavity structure is installed to stimulate the resonant-enhanced coherent plasmon excitation. ${ }^{32,39}$
AC-DGG HEMTs were designed and fabricated using InAlAs/InGaAs/InP materials [see Fig. 4(b)]. Two grating gates G1 and G2 were formed with 70-nm-thick Ti/Au/Ti by a standard lift-off process. An asymmetric factor, the ratio of the interfinger spaces, $d_{1} / d_{2}$, was fixed to be 0.5 . The grating gates $\mathrm{G} 1$ with narrower fingers $L_{g 1}$, serving plasmon cavity gates, were designed to be chirped ranging from 215 to $430 \mathrm{~nm}$. The design parameters are summarized in Table 1. After processing the AC-DGG HEMT, a high-Q vertical cavity with a high finess of $\sim 60$ was formed with a $4.4-\mu$ m-thick transparent PW1500 resist as a low-loss buffer layer and a 70-nm-thick indium tin oxide (ITO) mirror coat on top and a 100-nm Ti/Au coat on the polished back surface [see Fig. 4(b)]. Its fundamental resonant frequency (free spectral range of the Fabry-Perot modes) was designed to be $65 \mathrm{GHz}$.

The fabricated HEMTs exhibit normal dc transfer characteristics with good pinch-off and gate modulations for both G1 and G2 with threshold levels of -1.1 and $-0.9 \mathrm{~V}$, respectively. We conducted $\mathrm{THz}$ spectroscopic measurements for the fabricated devices using a Fourier-transform far-infrared (FTIR) spectrometer and a 4.2-K-cooled $\mathrm{Si}$ composite bolometer. The gate bias for the plasmon cavities $V_{g 1}$ was fixed at $0 \mathrm{~V}$. Material- and structure-dependent emission spectra at $290 \mathrm{~K}$ are plotted in Fig. 5(a) in comparison with previously reported GaAs-based and InP-based S-DGG HEMTs. ${ }^{39}$ The drain bias was set so as to equal the voltage drop along a unit DGG section among all the devices. All three S-DGG HEMTs SG1, SG2, and SP1 exhibit weak Fabry-Perot-modes emission and intense broadband emission as a background originated from the thermal excitation of broadband nonresonant plasmons by hot electrons. ${ }^{2-7}$ On the contrary, superiorities of the A-DGG structure and InP-based materials with higher electron mobility $\left[\sim 11,000 \mathrm{~cm}^{2} /(\mathrm{Vs})\right]$ result in intense Fabry-Perot-modes emission and weakened background emission. The identification of the plasmon modes in the emission spectra is based on (1) designed structure dimensions and material properties that determine the mode frequencies, and (2) dependence of the spectral peak intensity on $V_{d s}$. If the frequency of a spike in the emission spectrum corresponds to a plasmon resonant mode, its peak intensity will increase with increasing $V_{d s}$, thus promoting the instability. 2-D electron density distribution is modulated by $V_{d s}$; the plasmon mode frequencies become slightly lower with increasing $V_{d s}$. This mode-frequency shift might mask the increase in peak intensity if the mode frequency is detuned from the Fabry-Perot mode. The fundamental plasmon mode of the device ACP1 has a frequency of $\sim 1.2 \mathrm{THz}$. 


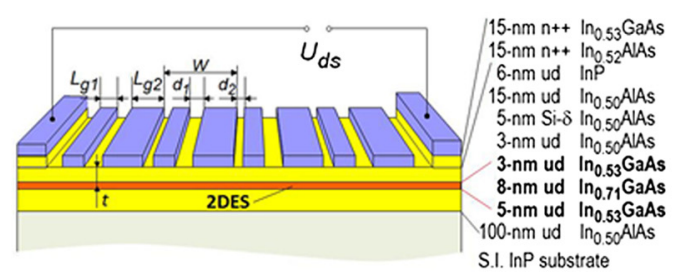

(a)

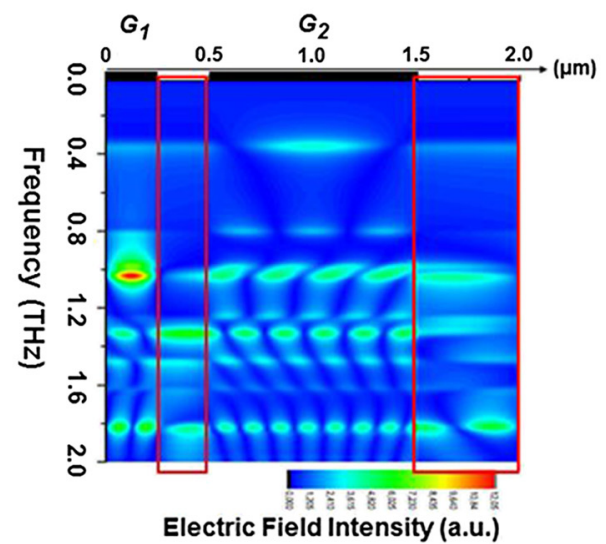

(c)

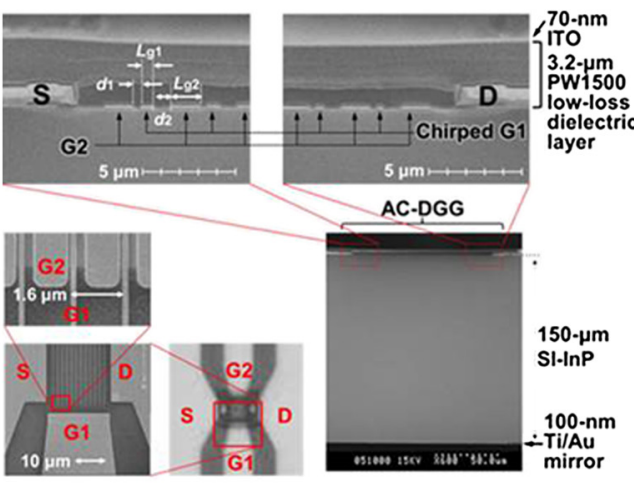

(b)

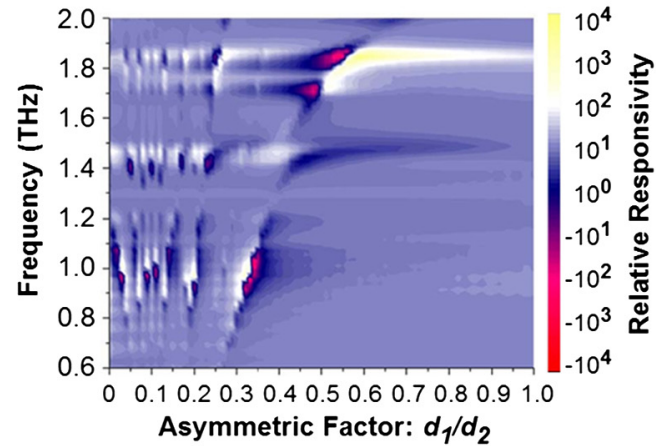

(d)

Fig. 4 (a) Schematic view of an asymmetric DGG (A-DGG) HEMT THz emitter. (b) Cross-sectional and top SEM images for a fabricated asymmetric chirped DGG (AC-DGG) HEMT with a resonant-enhanced vertical cavity structure. $L g 1=215$ to $430 \mathrm{~nm}, L g 2=1.6 \mu \mathrm{m}, d 1=200 \mathrm{~nm}$, $d 2=400 \mathrm{~nm}$. (c) Simulated THz electric field distribution underneath a unit A-DGG cell. Red squares show the asymmetric areas. $L g 1=250 \mathrm{~nm}, L g 2=1.0 \mu \mathrm{m}, d 1+d 2=750 \mathrm{~nm}, W=2.0 \mu \mathrm{m}$. Electron density under the gate $\mathrm{G} 1$ and $\mathrm{G} 2$ are $2.5 \times 1012 \mathrm{~cm}^{-2}$ and $2.5 \times 1011 \mathrm{~cm}^{-2}$, respectively. High electric field concentration with the fundamental plasma frequency of $1 \mathrm{THz}$ under $\mathrm{G} 1 \mathrm{can}$ be confirmed. (d) Simulated responsivity enhancement factor for an A-DGG HEMT under drain-unbiased conditions as a function of the asymmetry factor $d 1 / d 2$ with respect to that for a S-DGG HEMT for a dc drain bias current density of $0.1 \mathrm{~A} / \mathrm{m}$ (after Ref. 39).

Table 1 Design parameters of fabricated devices.

\begin{tabular}{|c|c|c|c|c|}
\hline Sample \# & SG1 & SG2 & SP1 & ACP1 \\
\hline Materials & \multicolumn{2}{|c|}{$\operatorname{lnGaP} / \operatorname{In}_{0.2} \mathrm{GaAs} / \mathrm{GaAs}$} & \multicolumn{2}{|c|}{$\operatorname{InAIAs} / \operatorname{InGaAs} s_{x} / \operatorname{In} \mathrm{P}^{a}$} \\
\hline DGG type ${ }^{b}$ & & S-DGG & & AC-DGG \\
\hline Cavity $^{\mathrm{C}}$ & Lo Q & High Q & Lo $Q$ & High Q \\
\hline FSR & $145 \mathrm{GHz}$ & $65 \mathrm{GHz}$ & $65 \mathrm{GHz}$ & $65 \mathrm{GHz}$ \\
\hline$L_{g 1}(\mathrm{~nm})$ & 100 & 100 & 100 & $215-430$ \\
\hline$d_{1} / d_{2}(\mathrm{~nm})$ & & $100 / 100$ & & $200 / 400$ \\
\hline$L_{g 2}(\mathrm{~nm})$ & 1800 & 850 & 800 & 1600 \\
\hline \# of fingers $\mathrm{G} 1 / \mathrm{G} 2$ & $37 / 38$ & $30 / 31$ & $30 / 31$ & $8 / 9$ \\
\hline Active area $\left(\mu \mathrm{m}^{2}\right)$ & $75 \times 75$ & $30 \times 75$ & $30 \times 75$ & $20 \times 20$ \\
\hline Ref. & {$[33-34]$} & - & [33] & [39-44] \\
\hline
\end{tabular}

${ }^{a} x=0.53 / 0.7 / 0.53$ (composite channel)

bS: Symmetric, AC: Asymmetric and chirpad

'Lo Q: Au mirror coat on back surface only 
From the observed emission spectra, the first, third, and fifth modes could be identified as designated with arrows in Figs. 5(b) and 5(c). The highest peak intensity is observed at $\sim 6.2 \mathrm{THz}$ with a sharp linewidth of $\sim 1.23 \mathrm{~cm}^{-1}$ $(\sim 37 \mathrm{GHz})$ corresponding to the fifth plasmon mode. Figure 5(b) plots the dependence of the emission spectra on $V_{d s}$ under an optimal $V_{g 2}$ condition $\left(V_{g 2}=-0.55 \mathrm{~V}\right){ }^{39,44}$ When $V_{d s}$ decreases from 1.0 to $0.4 \mathrm{~V}$, both DS and RSS instabilities are weakened so that the stimulated coherent emission is suppressed. This may increase the hot plasmon-originated spontaneous broadband emission and thus the monochromaticity on emission spectra is degraded. When the temperature cools down to $190 \mathrm{~K}$, hot plasmons-originated residual spurious modes are suppressed, resulting in intense monochromatic emission at the first (at $1.2 \mathrm{THz}$ ) and third (at $3.6 \mathrm{THz}$ ) plasmon modes whose frequencies match to adjacent Fabry-Perot modes, respectively, as shown in Fig. 5(b). The emission peak at the fifth (at $6.1 \mathrm{THz}$ ) plasmon mode disappears, which is considered to be caused by the temperature dependence of the 2-D electron density in the plasmon cavities reflecting frequency detuning of the plasmon modes. Simulated time evolution of the instantaneous electric field $\left(E_{x}\right)$ distributions under a constant sinusoidal plasmon excitation at $3.6 \mathrm{THz}$ assures the cooperative oscillation of all the plasmon cavities underneath the subgrating finger G1 as shown in Fig. 5(c), supporting the superradiant THz emission. The output power of sample ACP1 is estimated to be of the order of 0.1 to $1 \mu \mathrm{W}$.

\section{THz Detection Using 2-D Plasmons in HEMTs}

\subsection{Theory}

Detection of $\mathrm{THz}$ radiation by plasmonic nonlinearities in a 2-D electron channel of a field-effect transistor (FET) was originally proposed by Dyakonov and Shur. ${ }^{19}$ Resonant (frequency selective) ${ }^{20,21,24-26}$ as well as nonresonant (broadband) $)^{22,23,26}$ plasmonic detectors have been studied. The frequencies of the plasmon resonances in the FET channel with asymmetric boundary conditions are given by ${ }^{19}$

$\omega_{p}=n \frac{\pi}{2 L_{g-e f f}} \sqrt{\frac{e^{2} N^{(0)} d}{m^{*} \varepsilon \varepsilon_{0}}}, \quad e N^{(0)}=\varepsilon \varepsilon_{0} \frac{V_{g}-V_{t h}}{d}$,

where $N^{(0)}$ is the equilibrium electron density in the channel, $d$ and $\varepsilon$ are the thickness and relative dielectric constant of the barrier layer, $\varepsilon_{0}$ is the dielectric constant in vacuum, $L_{g \text {-eff }}$ is the effective length of the gated 2-D electron channel (which is greater than a geometrical length of the gate, $L_{g}$, due to the electric-field fringing effect), $n$ is an integer, $e$ and $m *$ are the electron charge and electron effective mass, respectively, $V_{g}$ is the gate-to-source dc bias voltage, and $V_{\text {th }}$

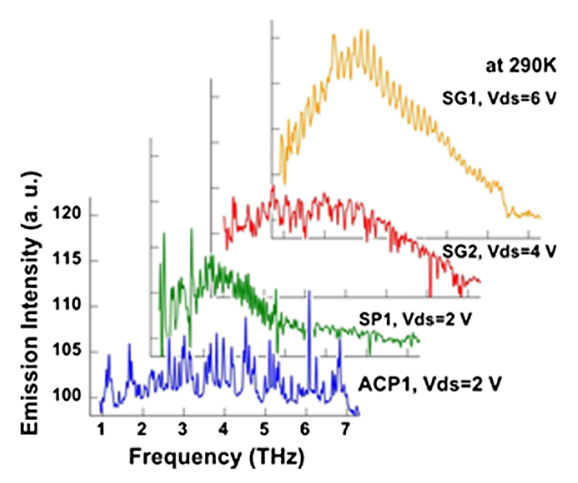

(a)

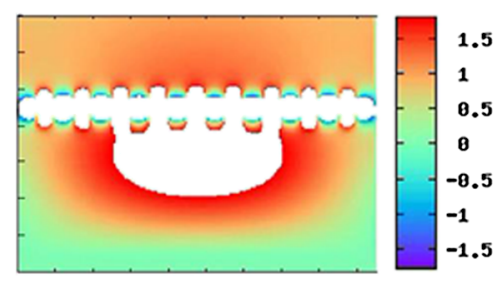

(c)

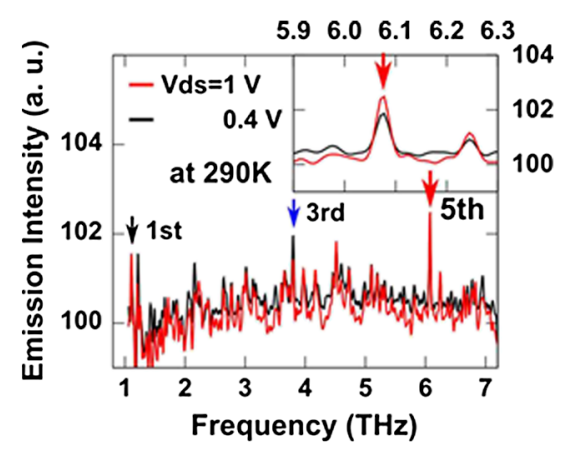

(b)

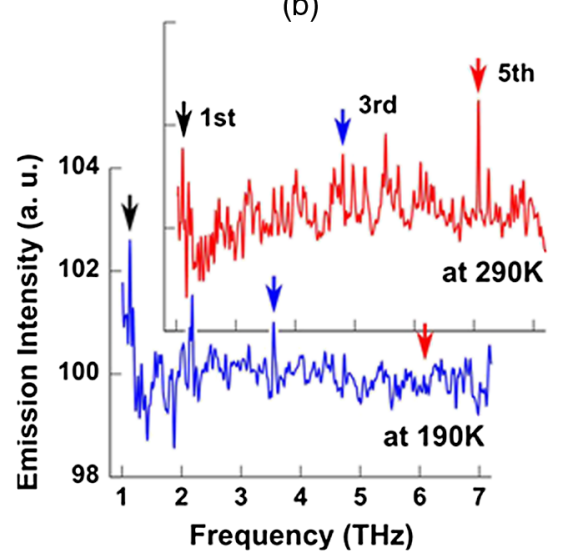

(d)

Fig. 5 (a) Measured emission spectra at $290 \mathrm{~K}$ for different material systems and DGG structures (after Ref. 39). (b) Measured emission spectra at different biases at $290 \mathrm{~K}$ (upper) and at different temperatures at optimal bias conditions $\left(V_{d s}=2 \mathrm{~V}, V_{g 1}=0 \mathrm{~V}, V_{g 2}=-0.5 \mathrm{~V}\right)$ (lower) of sample ACP1 (InP-based AC-DGG HEMT). Arrows designate the first, third, and fifth plasmon modes. Inset magnifies the spectral portions of the third and fifth mode (after Ref. 44). (c) Cross-sectional view of a simulated instantaneous electric field $\left(E_{x}\right)$ distributions under a constant sinusoidal plasmon excitation at 3.6 THz. All the plasmon cavities are cooperatively resonated in phase so that the uniform transverse radiation over the entire DGG area. 
is the threshold voltage of the FET. For an asymmetric boundary condition, only the plasmon modes with odd indices $n$ can be excited by the THz radiation normal to the FET-channel plane having the electric field component parallel to the channel direction, whereas the plasmon modes with even indices $n$ remain dark. Resonant detection takes place at $\mathrm{THz}$ frequencies $\omega=\omega_{p}$ for high-quality factors of the plasmon resonance such as $\omega_{p} \tau>1$, where $\tau$ is the electron momentum relaxation time. The nonresonant plasmonic detection takes place when the relaxation time is low (low mobility case) or equilibrium electron density in the FET channel decreases so that the inequality becomes valid for $V_{g} \rightarrow V_{\text {th }}$.

The hydrodynamic motion of the plasma wave kinetics yields a nonlinear plasma wave current component as the product of the local electron density and local electron velocity, both of which are perturbed by incoming $\mathrm{THz}$ radiation, giving rise to a quadratic rectification component in the current time-integral as shown in Fig. 6. This is the fundamental principle of the $\mathrm{THz}$ detection using the plasma wave dynamics. ${ }^{19,22}$ In the open circuit condition, the generated (rectified) photocurrent is transformed to a dc voltage component leading to photovoltaic signal $\partial U$. By solving Eqs. (1) and (2) under pertinent source-terminated and drainopened boundary conditions, $\partial U$ is derived as follows: ${ }^{19}$

$$
\left.\begin{array}{rl}
\partial U & \simeq \frac{U_{a}^{2}}{4 U_{0}^{2}} \frac{\omega_{0}^{2}}{\left(\omega^{2}-\omega_{0}^{2}\right)+\left(\frac{1}{2 \tau}-\frac{v_{d}}{L}\right)^{2}} \\
& \asymp \frac{U_{a}^{2}}{U_{0}^{2}}\left(\frac{s \tau}{L}\right)^{2} \frac{1}{4\left(\omega-n \omega_{0}\right)^{2} \tau^{2}+1}, \quad \text { for } \omega \tau \gg 1(\text { resonant mode }) \\
& \asymp 1+\frac{2 \omega \tau}{\sqrt{1+\omega^{2} \tau^{2}}}, \quad \text { for } \omega \tau \ll 1(\text { non-resonant mode })
\end{array}\right\} .
$$

where $s$ is the plasma wave velocity, $\omega_{0}$ is the fundamental plasmon resonant frequency, $n$ is the harmonic number, $\tau$ is the electron momentum relaxation time, $U_{a}$ is the voltage amplitude of the incident $\mathrm{THz}$ wave, and $U_{0}$ is the $\mathrm{dc}$ gate-to-channel potential.

As is mentioned in Sec. 2.2, we propose an A-DGG structure as shown in Fig. 4, which also provides ultrahighsensitive $\mathrm{THz}$ detection. ${ }^{37-40}$ This is because the unit cell of the A-DGG structure can create a strong built-in asymmetric field. The $\mathrm{THz}$ photoresponse dramatically increases when the parts of the 2-D channel under the fingers of one of the two subgratings are depleted.

The A-DGG structure can greatly enhance the asymmetry of the cavity boundaries by applying different gate voltages $V_{g 1}$ and $V_{g 2}$ to the two different subgratings of the A-DGG. Due to the asymmetry, the impedances at both ends from the 2-D plasmon cavity are different. Thus, the photocurrent generated in 2-D plasmon by the $\mathrm{THz}$ radiation performs unilateral transport, resulting in high photovoltaic signal

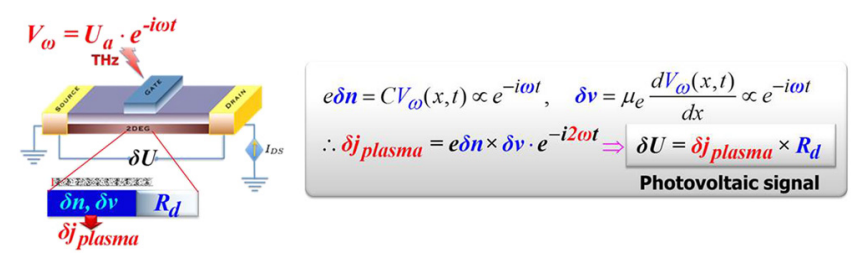

Fig. 6 Principle of rectification and detection of $\mathrm{THz}$ radiation by means of hydrodynamic nonlinearity of 2-D plasmons. at each cell. These signals at every 2-D plasmon cavities are summed up, leading to an effective photovoltaic signal between the drain and source. ${ }^{37}$

\subsection{Experiments}

The device under characterization is identical to the InAlAs/ InGaAs/InP A-DGG HEMTs shown in Fig. 4 except for the lack of a vertical cavity [seen in the lower right panel of Fig. 4(b)]. The A-DGG HEMTs have been designed and fabricated using InAlAs/InGaAs/InP material system. ${ }^{37}$ The geometrical parameters of the A-DGG HEMTs are summarized in Table 2. Room temperature $\mathrm{THz}$ photovoltaic measurements were conducted with fabricated devices. The devices exhibit normal gate modulation operation on their drain-source current-voltage characteristics. ${ }^{37}$ For G1 and G2, the threshold voltage $V_{\text {th1 }}$ and $V_{\text {th2 }}$ are -1.2 and $-1.0 \mathrm{~V}$, respectively.

We conducted room temperature $\mathrm{THz}$ photovoltaic measurements with the fabricated detectors. Monochromatic $\mathrm{THz}$ pulsed waves with frequencies from 1 to $3 \mathrm{THz}$ from a ring-cavity $\mathrm{THz}$ parametric oscillator source are focused on the detector by a Tsurupica ${ }^{\mathrm{TM}}$ lens. ${ }^{37-40}$ The photovoltaic signal was observed as a $V_{d s}$ fluctuation with a lock-in technique. The responsivity was estimated as $R_{v}=$ $\Delta U S_{t} / P_{t} S_{d}$, where $\Delta U$ is the THz-radiation-induced dc drain voltage, $P_{t}$ is the total power of the source on the detector plane, $S_{t}$ is the radiation beam spot area, and $S_{d}$ is the active area of the detector. Figure 7(a) shows the measured responsivity of detector \# 2-4 at $1 \mathrm{THz}$ under zero- $V_{d s}$ condition as a function of gate voltage swing $\left(V_{g 1,2}-V_{\mathrm{th}}\right)$; dc voltage of G1: $V_{g 1}\left(\mathrm{G} 2: V_{q 2}\right)$ is swept, while $V_{g 2}\left(V_{g 1}\right)$ is floated (biased at $0 \mathrm{~V}){ }^{37}$ The best result with $R_{v}=$ $2.2 \mathrm{kV} / \mathrm{W}$ was obtained when sweeping $V_{g 1}$ to the threshold $V_{\text {th }}{ }^{37,38}$ It should be noted that even at higher frequencies, relatively high responses are obtained with detectors \# 2-3 having a shorter $L_{g 1}$. As seen in the inset of Fig. 7(a), the responsness monotonically decreases from $1.7 \mathrm{kV} / \mathrm{W}$ at $1 \mathrm{THz}$ to $0.52 \mathrm{kV} / \mathrm{W}$ at $2 \mathrm{THz}$. All these values are, to the best of the authors' knowledge, the best ever reported at these frequencies. Figure 7(b) shows the measured responsiveness of detector \# 2-3 at $1.5 \mathrm{THz}$ under $V_{d s}$-biased con-

\begin{tabular}{|c|c|c|c|}
\hline Sample \# & $1-1$ & $2-3$ & $2-4$ \\
\hline Materials & \multicolumn{3}{|c|}{$\operatorname{lnAIAs} / \operatorname{lnGaAs} s_{x} / \ln P^{a}$} \\
\hline DGG type ${ }^{b}$ & \multicolumn{3}{|c|}{ AC-DGG } \\
\hline$L_{g 1}(n m)$ & 200 & $215-430$ & $400-705$ \\
\hline$d_{1} / d_{2}(\mathrm{~nm})$ & $200 / 400$ & $200 / 400$ & $400 / 800$ \\
\hline \# of fingers $\mathrm{G} 1 / \mathrm{G} 2$ & $8 / 9$ & $8 / 9$ & $6 / 7$ \\
\hline Ref. & [40] & [37-39] & [37-39] \\
\hline
\end{tabular}
ditions. On increasing $V_{d s}$ from 0 to $0.4 \mathrm{~V}$, the maximum

Table 2 Design parameters of fabricated A-DGG HEMTs. 


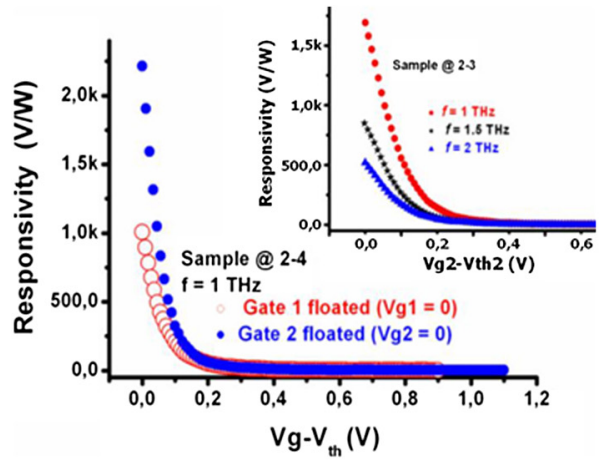

(a)

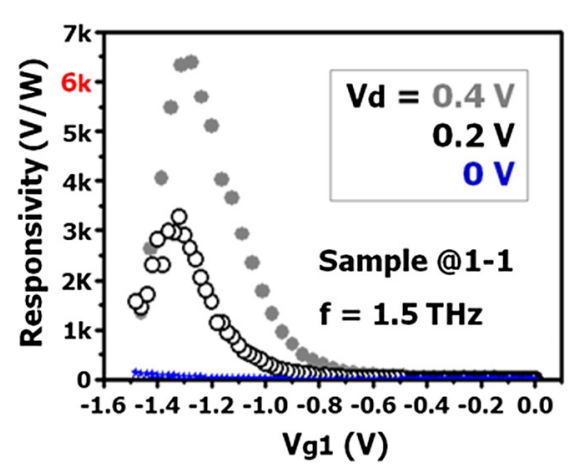

(b)

Fig. 7 (a) Responsivity of detector \# 2-4 as a function of the gate swing voltage $\left(V_{g 1}-V_{\text {th1 } 1}\right.$ or $\left.V_{g 2}-V_{\text {th2 } 2}\right)$ at 1 THz under drain-unbiased condition. Inset shows responsivities of detector \# 2-3 at 1, 1.5, and $2 \mathrm{THz}$ as a function of the gate swing voltage $V_{g 2}-V_{\text {th2 }}$ when G1 is floated. The responsivities become higher with lowering the gate voltages (taking the maximum at the threshold) (after Ref. 37). (b) Responsivity of detector \# 1-1 as a function of the gate swing voltage at $1.5 \mathrm{THz}$ radiation under drain-biased conditions (after Ref. 40).

responsiveness increases to $6.4 \mathrm{kV} / \mathrm{W}$, which is, to the best of the authors' knowledge, the record responsiveness of fast-response detectors at $300 \mathrm{~K} .{ }^{40}$ The photovoltaic signal increases on decreasing the gate bias and takes a peak value when the gate bias is approaching the threshold level for all the photoresponses. It is expected to turn down and drop when the gate bias decreases to the subthreshold region as was measured for S-DGG HEMTs ${ }^{35}$ as well as standard single-gate HEMTs. ${ }^{22}$ Very recently, a physical model that explains this photoresponse dependence on gate bias has been developed. ${ }^{45}$

Figure 8 shows noise equivalent power (NEP) for detectors (a) \# 2-4 and (b) \# 2-3, as a function of $V_{g 1,2}$ under drainunbiased condition. ${ }^{37}$ The detector \# 2-3 exhibits extremely low NEP with the minimal value $15 \mathrm{pW} / \mathrm{Hz}^{0.5}$ at $1 \mathrm{THz}$. These values are lower than those of commercial room temperature $\mathrm{THz}$ detectors such as Golay cells $\left(200 \mathrm{pW} / \mathrm{Hz}^{0.5}\right)$ (Ref. 46) or Schottky barrier diodes $\left(100 \mathrm{pW} / \mathrm{Hz}^{0.5}\right){ }^{36}$ The NEP value is proportional to the square root of the drain resistance $R_{d}^{0.5}$ and the inverse responsivity $R_{v}^{-1}$. On decreasing the gate swing voltage $\left(V_{g 1,2}-V_{\text {th }}\right)$, the responsivity becomes larger in the range $\left(V_{g 1,2}-V_{\text {th }}\right)<0.2 \mathrm{~V}$ (see Fig. 8), resulting in the decrease of the NEP value. On the other hand, near the threshold condition $\left(V_{g 1,2}-V_{\text {th }}\right) \sim 0$, the drain resistance becomes much larger because of the pinch-off, resulting in an increase of the NEP value. As a result, the NEP value becomes minimal around the midpoint $\left(\left(V_{g 1,2}-V_{\text {th }}\right) \sim\right.$ $-0.1 \mathrm{~V}$. This trend is seen for all the A-DGG HEMT devices and bias conditions due to very similar characteristics of drain resistance and responsiveness for both gates at every frequency measured.

The detection performance at a low frequency of $292 \mathrm{GHz}$ was also characterized at low temperatures ranging from 50 to $125 \mathrm{~K}$ (see Fig. 9). ${ }^{44}$ By decreasing the temperature, one can see a peak appearing as a shoulder and narrowing on the detection curves. This peak is identified as a gated plasmon resonance and its frequency can be estimated as $\delta f=$ $(f / 2) *\left[\delta V_{g 1} /\left(V_{g 1}-V_{\text {th1 }}\right)\right]$, where $f$ is the incident frequency and $V_{\text {th } 1}=-1.2 \mathrm{~V}$. This width is found to vary from 279 to $100 \mathrm{GHz}$ when decreasing the temperature. The observed shrinking of the resonance peak can be explained by an increase of plasma waves damping time $\tau$ via an increase of carrier's mobility. The quality factor of the resonances' linewidth is experimentally determined by $\delta f / f=$ 3 from which the plasmon decay time is estimated to be $\sim 2$ ps. This quality factor should reflect on the response speed of the plasmon-resonant. Therefore, very fast speed $\mathrm{THz}$ detection beyond tens of Gbit/s could be feasible. Its precise characterization is a future subject of study. The device response speed is characterized indirectly by the resonant-mode detection. Figure 9 also shows calculations of channel resistivity $R$ and the difference induced in drain

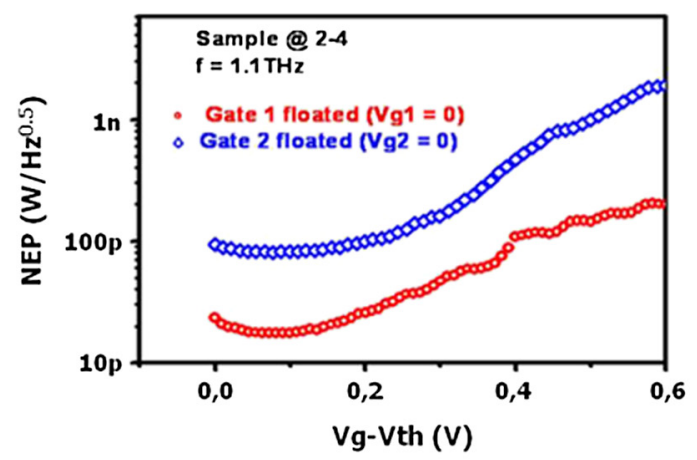

(a)

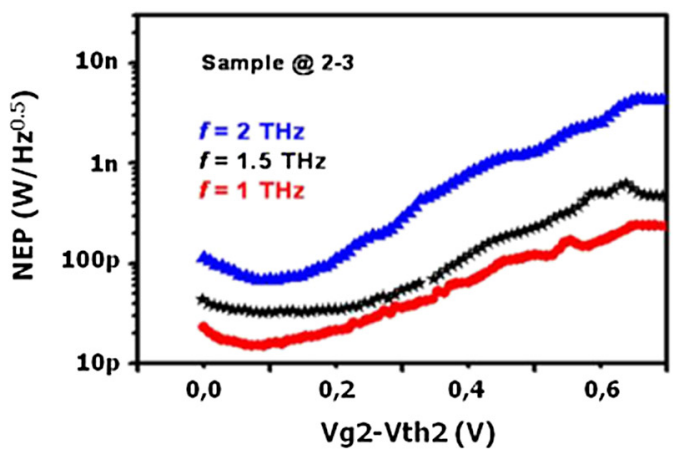

(b)

Fig. 8 Measured noise equivalent power for (a) sample \# 2-4 at $1 \mathrm{THz}$ as functions of the gate swing voltages $V_{g 1}-V_{\text {th1 }}$ and $V_{g 2}-V_{t h 2}$ and (b) sample \# 2-3 at 1, 1.5, and $2 \mathrm{THz}$ as a function of the gate swing voltage $V_{g 2}-V_{\text {th2 }}$. The noise equivalent power becomes minimum at $V_{g 1,2}-$ $V_{\text {th }} \sim 0.1 \mathrm{~V}$ (after Ref. 37 ). 


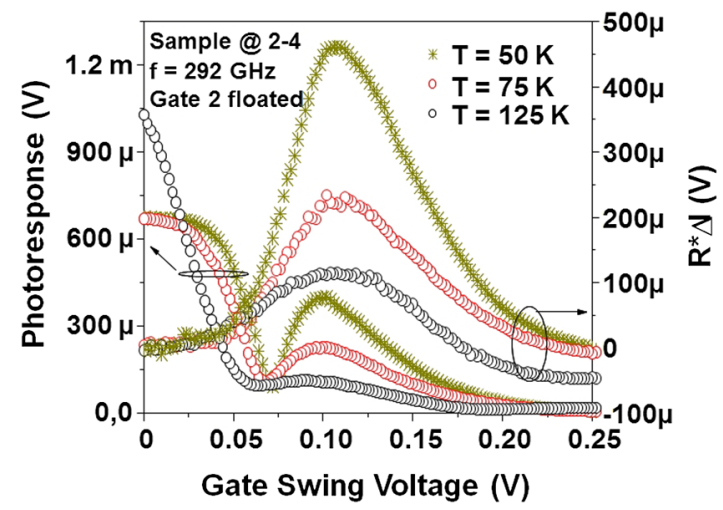

Fig. 9 Measured photoresponse at $292 \mathrm{GHz}$ for different temperatures as a function of gate voltage swing (left) and calculated curves $R * \Delta /$ (right), where $\mathrm{R}$ is the channel resistivity, $\Delta /$ (I with radiation $-\mathrm{I}$ without radiation) is the difference induced in drain current by the incoming $\mathrm{THz}$ radiation. (after Ref. 40).

current by the incoming THz radiation $\Delta I$ (I with radiationI without radiation). The $R * \Delta I$ curves (right axis) consistently shows the resonant peak obtained from experiments.

\subsection{Discussion}

Utilizing the excitation of 2-D plasmons is a promising approach for both emission and detection of $\mathrm{THz}$ radiation. Recently, there were several outstanding works published on $\mathrm{THz}$ plasmonic detection by nanowire single-gate FETs ${ }^{47}$ as well as graphene-channel single-gate FETs. ${ }^{48}$ A comprehensive review of all these single-gate plasmonic nanotransistor detectors is given in a recent paper by Knap et al. ${ }^{49}$ The DGG HEMT detectors can potentially offer better figures of merit because of their following important advantages against all these single-gate FET detectors.

1. The DGG-HEMT THz detector does not need supplementary antenna elements because the grating gate itself serves as an effective coupler between $\mathrm{THz}$ radiation and plasmons in the HEMT channel. On the contrary, a single-gate plasmonic FET weakly couples to $\mathrm{THz}$ radiation and hence needs supplementary antenna elements.

2. Asymmetry needed for obtaining strong detection signal is introduced into each unit cell of the DGG structure in a controlled way by using monolithically integrated technology. Therefore, all unit cells operate coherently, yielding the strong cumulative resultant detection response.

3. The DGG-HEMT combines useful properties of both resonant and nonresonant $\mathrm{THz}$ plasmonic detectors. Plasmons (also nonresonant plasmons) are effectively excited in undepleted portions of the entire DGGHEMT channel, while strongly depleted (hence highly resistive) portions of the DGG-HEMT channel under the biased subgrating of the DGG serve for effective readout of the photovoltaic detection signal.

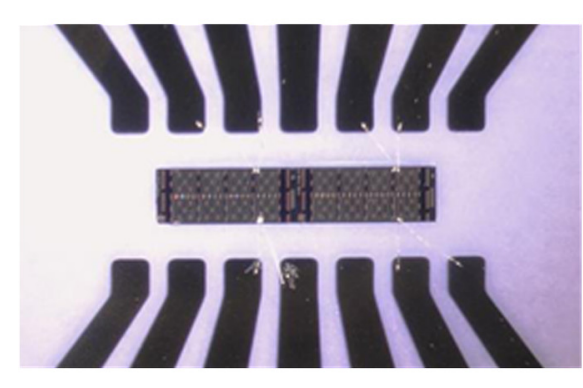

(a)

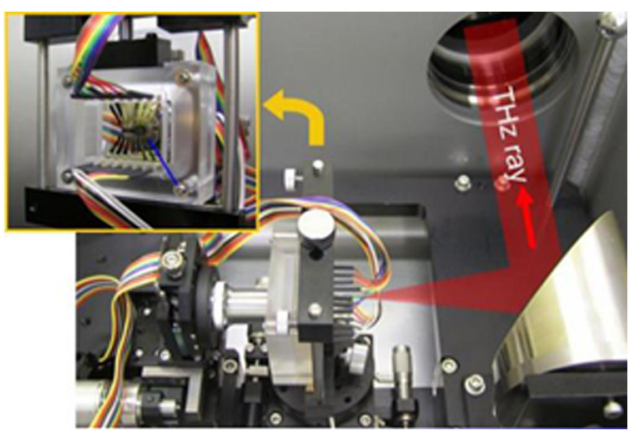

(b)

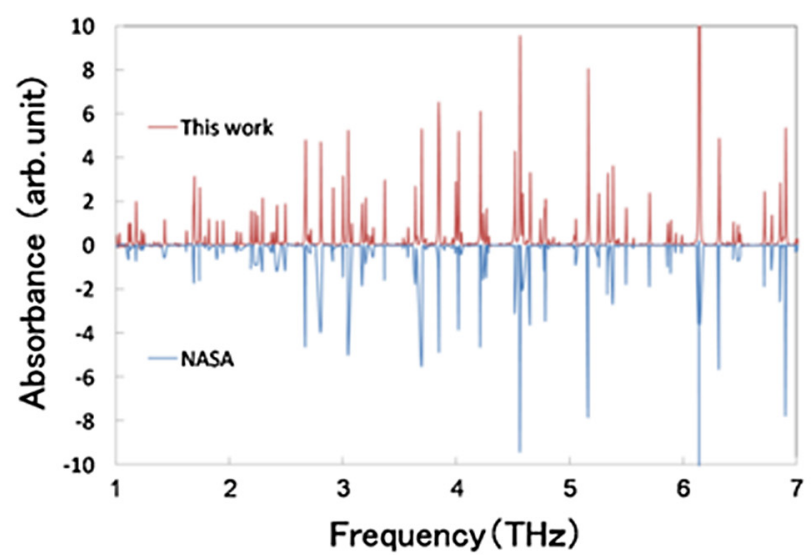

(c)

Fig. 10 (a) $2 \mathrm{~mm} \times 10 \mathrm{~mm}$ die (accommodating 32 independent emitter devices) mounted on a quartz substrate. Two emitters are wire-bonded with metalized patterns on the substrate for electrical connection. (b) Emitter chip mounted on a sample holder installed in the chamber of the FTIR spectrometer equipment. (c) Measured absorption spectrum of atmospheric water vapor in comparison with the data provided by NASA (after Ref. 35). 
All these advantageous features combined together result in a huge THz photovoltaic response of the DGG-HEMT detectors as mentioned above.

\section{Applications to Nondestructive $\mathrm{THz}$ Spectroscopy}

\subsection{2-D Plasmon Emitters for Broadband THz Light Sources in Fourier Transform $\mathrm{THz}$ Spectroscopy}

The plasmon resonant A-DGG-HEMT emitter SG-1 shown in Fig. 5(a) was introduced into the FTIR system in place of the mercury lamp and its applicability as a broadband $\mathrm{THz}$ light source was examined. The detector was a $4.2-\mathrm{K}$ cooled Si composite bolometer whose absolute detection power was not calibrated so that we could characterize the relative intensity spectra. Atmospheric water vapor and several sugar groups, whose identical absorption/transmis- sion spectra coexist in the emission spectral range available from the emitter, are chosen as samples under measurement. First, the atmospheric water-vapor absorption was measured. The experimental procedure was as follows: first, we measured the emission spectra in vacuum inside the spectrometer as the reference. Then, the atmospheric air was introduced into the spectrometer and we performed the actual measurement. The obtained result was normalized to the reference data. The FTIR system (Fig. 10) shows the measured absorption spectrum of the water vapor. The result ranging from 1 to $6.5 \mathrm{THz}$ coincides well with the spectrum data provided by NASA. ${ }^{34}$

Next, we measured the transmission spectra of two different types of sugar groups: honey and maple syrup, both of which contain featured spectrum in the $\mathrm{THz}$ region. Figures 11(a) and 11(b) plot the results measured by using the plasmon-resonant emitter (this work) and by using a

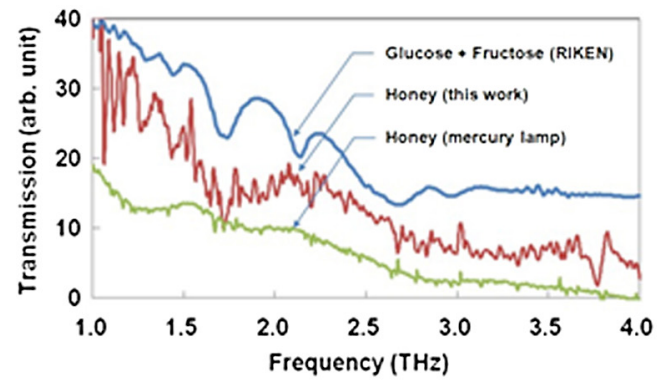

(a)

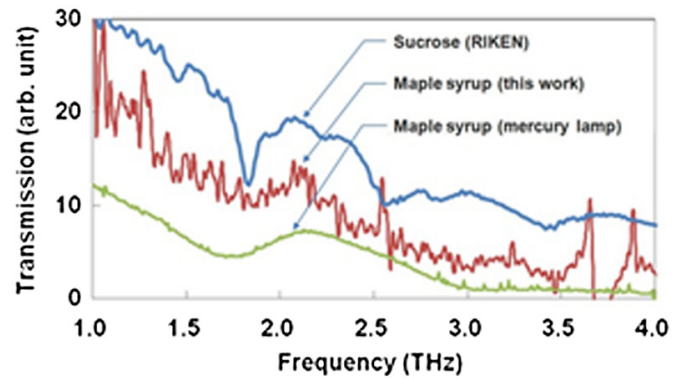

(b)

Fig. 11 Transmission spectra of sugar groups measured by using a plasmon-resonant emitter (this work) and by using a high-pressure mercury lump, and their main ingredient(s) provided by RIKEN. (a) Honey versus glucose and fructose. (b) Maple syrup versus sucrose (after Ref. 35).

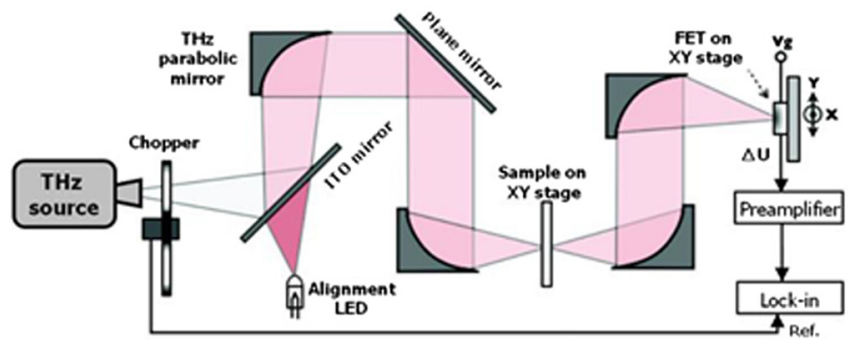

(a)
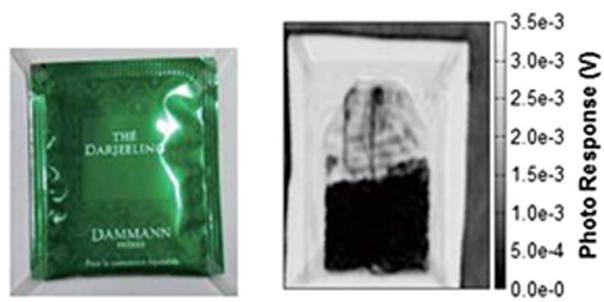

(c)

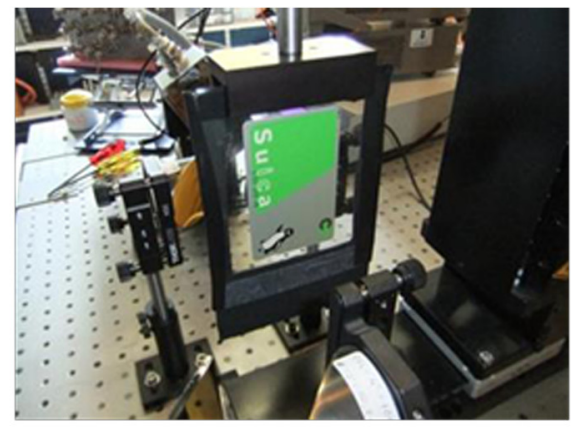

(b)
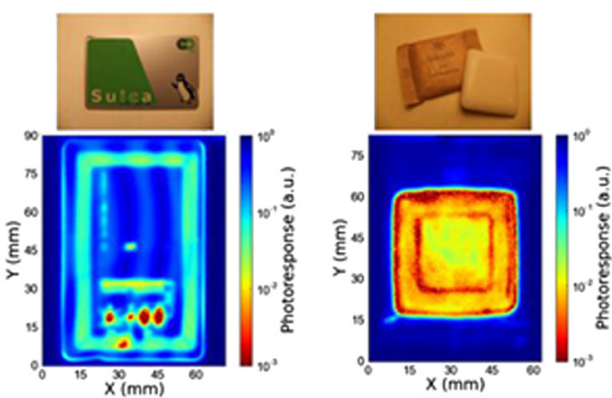

(d)

Fig. 12 Imaging setup and results using an InP-based S-DGG and A-DGG HEMTs. (a) Experimental setup for sub-THz imaging experiments. (b) Photo image of the sample holder of the imaging setup. (c) A tea bag measured by using an S-DGG HEMT. Left: photo image; right: THz image (after Ref. 38). (d) Photo images and THz imaging measured by using an A-DGG HEMT. Left: an IC card; right: a soap bar (after Ref. 39). 
high-pressure mercury lump, and their main component(s) provided by RIKEN. ${ }^{34}$ The measured samples-honey and maple syrup-were in liquid form, but the components of sugar groups measured by RIKEN were dry pellets. Thus, comparison of the absorption peak points among them does not make sense. However, the molecular structures may reflect on the overall spectral shape. They clearly exhibit identical spectral features for both materials. The major bumps measured for each spectrum correspond fairly well to those for the main components for each: (a) honey versus glucose and fructose and (b) maple syrup versus sucrose. Other minor part of the ingredients and/or impurities may also perturb the spectral shape. Compared with a high-power mercury lamp, the plasmon-resonant microchip emitter yields higher noise on the spectra due to weak emission intensity. Further improvements on its emission power will be feasible as is described before, which enables the device to be a promising candidate as a new $\mathrm{THz}$ light source.

\subsection{2-D Plasmon Detectors for THz Imaging}

A $\mathrm{THz}$ imaging experiment was carried out for several DGG HEMT detectors using the experimental setup shown in Figs. 12(a) (Ref. 38) and 12(b) (Ref. 39). The imaging results are shown in Figs. 12(c) (Ref. 38) and 12(d) (Ref. 39). The samples under imaging are (c) a tea bag with an aluminum-coated plastic package (a 50-nm-thick aluminum being vacuum-evaporated onto the plastic film) and (d) an IC card and a soap bar. As seen in Fig. 12(c), the $\mathrm{THz}$ radiation can transit through the aluminum-coated plastic package so that it could successfully identify the contents of the tea leaves inside the tea bag. As seen in Fig. 12(d), measured results clearly demonstrate the imaging of hidden substances of circuit/ antenna components inside of the IC card and the thickness of the soap bar with a nice contrast in a submm resolution.

\section{Conclusion}

Recent advances in emission and detection of $\mathrm{THz}$ radiation using 2-D plasmons in semiconductor nanoheterostructures for nondestructive evaluations were reviewed. The Dopplershift effect of the plasma wave velocity under an asymmetric plasmon cavity boundary and/or the spatial modulation of electron transit time in a sub-micrometer scale 2-D plasmon system with a nonuniform 2-D electron density distribution can promote the plasmon instability, resulting in self-oscillation of plasmons in the $\mathrm{THz}$ regime. The hydrodynamic nonlinearity of 2-D plasmons can rectify the incoming electromagnetic radiation, resulting in photovoltaic detection of $\mathrm{THz}$ radiation under an asymmetric 2-D plasmon cavity boundary. The device structure that can provide practical emission and detection performances was addressed and was based on an HEMT that incorporated an asymmetrically interdigitated DGGs structure. Excellent $\mathrm{THz}$ emission and detection performances including coherent, monochromatic emission beyond 1-THz range and an extremely high detection responsiveness of $2.2 \mathrm{kV} / \mathrm{W}$ at $1 \mathrm{THz}$ at $300 \mathrm{~K}$ were experimentally demonstrated by using InAlAs/ InGaAs/InP heterostructure material systems. Finally, several applications to nondestructive material evaluation based on $\mathrm{THz}$ imaging are demonstrated. The obtained results encourage us to proceed with further industrialization of these plasmonic $\mathrm{THz}$ devices.

\section{Acknowledgments}

The authors are grateful to M. Dyakonov, M. S. Shur, and S. A. Nikitov for valuable comments. This work has been supported by the JST-ANR Japan-France International Strategic Collaborative Research Program "Wireless communication using terahertz plasmonic nano ICT devices," the JSPSRFBR Japan-Russian Joint Research Program, the Russian Foundation for Basic Research (Grant \# 11-02-92101, 1202-93105, and 13-02-12070), and the Russian Academy of Sciences Program "Fundamentals of Nanostructure Technologies and Nanomaterials." The work was performed under the umbrella of the GDR-I project "Semiconductor sources and detectors for terahertz frequencies."

\section{References}

1. M. Tonouchi, "Cutting-edge terahertz technology," Nature Photon. 1(2), 97-105 (2007)

2. R. A. Hopfel, E. Vass, and E. Gornik, "Thermal excitation of twodimensional plasma oscillations," Phys. Rev. Lett. 49(22), 1667-1671 (1982).

3. D. C. Tsui, E. Gornik, and R. A. Logan, "Far infrared emission from plasma oscillations of Si inversion layers," Solid State Commun. 35(11), 875-877 (1980).

4. N. Okisu, Y. Sambe, and T. Kobayashi, "Far-infrared emission from two-dimensional plasmons in AlGaAs/GaAs heterointerfaces," Appl. Phys. Lett. 48(12), 776 (1986).

5. R. Hopfel et al., "Cyclotron and plasmon emission from twodimensional electrons in GaAs," Surf. Sci. 113(1-3), 118-123 (1982).

6. R. J. Wilkinson et al., "Plasmon excitation and self-coupling in a biperiodically modulated two-dimensional electron gas," J. Appl. Phys. 71(12), 6049-6061 (1992).

7. K. Hirakawa et al., "Far-infrared emission spectroscopy of hot twodimensional plasmons in Al0.3Ga0.7As/GaAs heterojunctions," Appl. Phys. Lett. 67(16), 2326 (1995).

8. M. Dyakonov and M. Shur, "Shallow water analogy for a ballistic field effect transistor: new mechanism of plasma wave generation by dc current," Phys. Rev. Lett. 71(15), 2465-2468 (1993).

9. W. Knap et al., "Terahertz emission by plasma waves in $60 \mathrm{~nm}$ gate high electron mobility transistors," Appl. Phys. Lett. 84(13), 2331 (2004).

10. J. Lusakowski et al., "Voltage tunable terahertz emission from a ballistic nanometer InGaAs/InAlAs transistor," J. Appl. Phys. 97(6), 064307 (2005).

11. N. Dyakonova et al., "Magnetic field effect on the terahertz emission, from nanometer InGaAs/AlInAs high electron mobility transistors," J. Appl. Phys. 97(11), 114313 (2005).

12. A. El Fatimy et al., "AlGaN/GaN high electron mobility transistors as a voltage-tunable room temperature terahertz sources," J. Appl. Phys. 107(2), 024504 (2010).

13. S. A. Mikhailov, "Plasma instability and amplification of electromagnetic waves in low-dimensional electron systems," Phys. Rev. B 58(3), 1517-1532 (1998).

14. P. Bakshi et al., "Plasmon-based terahertz emission from quantum well structures," Appl. Phys. Lett. 75(12), 1685 (1999).

15. R. Colombelli et al., "Far-infrared surface-plasmon quantum-cascade lasers at $21.5 \mu \mathrm{m}$ and $24 \mu \mathrm{m}$ wavelengths," Appl. Phys. Lett. 78(18), 2620 (2001)

16. A. Tredicucci et al., "Terahertz quantum cascade lasers-first demonstration and novel concepts," Semicond. Sci. Technol. 20(7), S222-S227 (2005).

17. V. Ryzhii, A. Satou, and M. Shur, "Plasma instability and terahertz generation in HEMTs due to electron transit-time effect," IEICE Trans. Electron. E89-C(7), 1012 (2006).

18. V. Ryzhii et al., "Mechanism of self-excitation of terahertz plasma oscillations in periodically double-gated electron channels," J. Phys.: Condens. Matter 20(38), 384207 (2008).

19. M. Dyakonov and M. Shur, "Detection, mixing, and frequency multiplication of terahertz radiation by two-dimensional electronic fluid," IEEE Trans. Electron. Dev. 43(10), 1640-1645 (1996).

20. F. Teppe et al., "Room-temperature plasma waves resonant detection of sub-terahertz radiation by nanometer field-effect transistor," Appl. Phys. Lett. 87(5), 052107 (2005).

21. V. M. Muravev et al., "Millimeter/submillimeter mixing based on the nonlinear plasmon response of two-dimensional electron systems," JETP Lett. 90(3), 197-201 (2009). 
22. W. Knap et al., "Field effect transistors for terahertz detection: physics and first imaging applications," J. Infrared Millim. Terahertz. Waves 30(12), 1319-1337 (2009).

23. W. Lisauskas et al., "Terahertz imaging with GaAs field-effect transistors," Electron. Lett. 44(6), 408-409 (2008)

24. A. El Fatimy et al., "Plasma wave field effect transistor as a resonant detector for 1 terahertz imaging applications," Opt. Commun. 282(15), 3055-3058 (2009).

25. S. Nadar et al., " Terahertz imaging using high electron mobility transistors as plasma wave detectors," Phys. Status Solidi C 6(12), 28552857 (2009).

26. W. Knap et al., "Field effect transistors for terahertz detection and emission," J. Infrared Millim. Terahertz. Waves 32(5), 618-628 (2011).

27. U. Lisauskas et al., "Rational design of highresponsivity detectors of terahertz radiation based on distributed self-mixing in silicon field-effect transistors," J. Appl. Phys. 105(11), 114511 (2009).

28. T. Tanigawa et al., "Enhanced responsivity in a novel AlGaN/GaN plasmon-resonant terahertz detector using gate-dipole antenna with parasitic elements," in 68th Device Research Conf. Dig., pp. 167-168, IEEE, New York (2010).

29. Y. F. Sun et al., "Room temperature GaN/AlGaN self-mixing terahertz detector enhanced by resonant antennas," Appl. Phys. Lett. 98(25), 252103 (2011).

30. F. Schuster et al., "Broadband terahertz imaging with highly sensitive silicon CMOS detectors," Opt. Express 19(8), 7827-7832 (2011).

31. V. Ryzhij, N. A. Bannov, and V. A. Fedirko, "Ballistic and quasiballistic transport in semiconductor structures," Sov. Phys.-Semicond. 18(5), 481-491 (1984)

32. T. Otsuji et al., "A grating-bicoupled plasma-wave photomixer with resonant-cavity enhanced structure," Opt. Express 14(11), 4815-4825 (2006).

33. T. Otsuji et al., "Emission of terahertz radiation from two-dimensional electron systems in semiconductor nano-and hetero-structure," J. Infrared Millim. Terahertz Waves 32(5), 629-645 (2011).

34. Y. Tsuda et al., "Application of plasmonic microchip emitters to broadband terahertz spectroscopic measurement," J. Opt. Soc. Am. B 26(9), A52-A57 (2009).

35. D. Coquillat et al., "Room temperature detection of sub-terahertz radiation in double-grating-gate transistors," Opt. Express 18(6), 6024-6032 (2010).

36. V. V. Popov et al., "Plasmonic terahertz detection by a double-gratinggate field-effect transistor structure with an asymmetric unit cell," Appl. Phys. Lett. 99(24), 243504 (2011).

37. T. Watanabe et al., "Ultrahigh sensitive plasmonic terahertz detector based on an asymmetric dual-grating gate HEMT structure," Solid State Electron. 78, 109-114 (2012).

38. T. Watanabe et al., "InP-and GaAs-based plasmonic highelectron-mobility transistors for room-temperature ultrahighsensitive terahertz sensing and imaging," IEEE Sensors J. 13(1), 89-99 (2013).

39. T. Otsuji et al., "Emission and detection of terahertz radiation using two-dimensional electrons in III-V semiconductors and graphene," IEEE Trans. Terahertz. Sci. Technol. 3(1), 63-72 (2013).

40. S. Boubanga-Tombet et al., "Asymmetric dual-grating gate InGaAs/ InAlAs/InP HEMTs for ultrafast and ultrahigh sensitive terahertz detection," in Device Research Conf. Dig., IV.A-10, pp. 169-170, IEEE, New York (2012)

41. Y. M. Meziani et al., "Room temperature terahertz emission from grating coupled two-dimensional plasmons," Appl. Phys. Lett. 92(20), 201108 (2008).

42. M. I. Dyakonov, "Boundary instability of a two-dimensional electron fluid," Semicond. 42(8), 984-988 (2008).

43. M. Dyakonov and M. S. Shur, "Current instability and plasma waves generation in ungated two-dimensional electron layers," Appl. Phys. Lett. 87(11), 111501 (2005).

44. T. Watanabe et al., "Plasmonic terahertz monochromatic coherent emission from an asymmetric chirped dual-grating-gate InAlAs/InGaAs/InP HEMT with highly asymmetric resonant cavities," in Int. Conf. on Optical Terahertz Science and Technology, Kyoto, Th3-26, OTST2013 Committee, Kyoto, Japan (4 April 2013).

45. M. Sakowicz et al., "Terahertz responsivity of field effect transistors versus their static channel conductivity and loading effects," J. Appl. Phys. 110(5), 054512 (2011).

46. OAD-7 Golay Detector Operating Manual, QMC Instruments Ltd., Cardiff, U.K. (2005)

47. M. S. Vitiello et al., "Room-temperature terahertz detectors based on semiconductor nanowire field-effect transistors," Nano Lett. 12 96-101 (2012).

48. L. Vicarelli et al., "Graphene field-effect transistors as roomtemperature terahertz detectors," Nature Mater. 11(10), 865-871 (2012).

49. W. Knap et al., "Nanometer size field effect transistors for terahertz detectors," Nanotechnology 24(21), 214002 (2013).

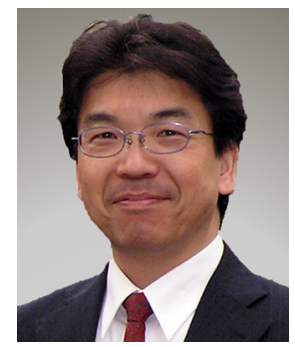

Taiichi Otsuji received his BS and MS degrees in electronic engineering from Kyushu Institute of Technology, Fukuoka, Japan, in 1982 and 1984, respectively, and his $\mathrm{PhD}$ degree in electronic engineering from Tokyo Institute of Technology, Tokyo, Japan, in 1994. From 1984 to 1999, he worked for NTT Laboratories, Kanagawa, Japan. In 1999, he joined Kyushu Institute of Technology as an associate professor, becoming a professor in 2002. Since 2005, he has been a professor at the Research Institute of Electrical Communication, Tohoku University, Sendai, Japan. His current research interests include terahertz electronic and photonic materials/devices and their applications. $\mathrm{He}$ is authored and coauthored more than 170 peer-reviewed journals. He is a senior member of the Institute of Electrical and Electronic Engineers (IEEE) and the Optical Society of America, and a member of the Institute of Electronics, Information, and Communication Engineers of Japan and the Japan Society of Applied Physics (JSAP). He was the recipient of the Outstanding Paper Award of the 1997 IEEE GaAs IC Symposium and has been awarded the IEEE Electron Device Society Distinguished Lecturer since 2013.

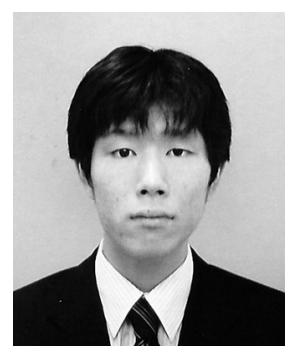

Takayuki Watanabe received his $\mathrm{BE}$ and ME degrees in electrical communication engineering from Tohoku University, Sendai, Japan, in 2009 and 2011, respectively. He is presently with the Research Institute of Electrical Communication, Tohoku University, Sendai, Japan and is working toward the Dr. Eng. degree. $\mathrm{He}$ is a student member of the JSAP.

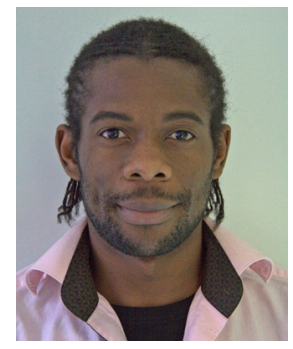

Stephane Albon Boubanga Tombet received his MS and $\mathrm{PhD}$ degrees in condensed matter physics from Montpellier 2 University in France in 2005 and 2008, respectively. From 2008 to 2009, he worked for Montpellier 2 University as a teacher and research assistant. From 2009 to 2011, he joined the Research Institute of Electrical Communication, Tohoku University, Sendai, as a postdoctoral researcher granted by the Japanese Society for the Promotion of Science (JSPS) for one year fellowship. Since 2012, he is working as a postdoctoral researcher at Los Alamos National Labs in New Mexico. His current research interests include terahertz electronic and photonic materials/devices and their applications. He has authored and coauthored more than 20 peer-reviewed journals.

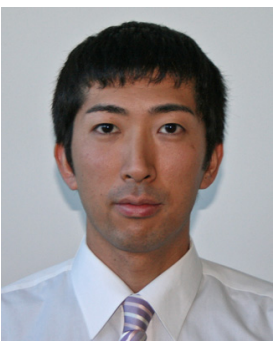

Akira Satou received his BS, MS, and doctor of computer science degrees from University of Aizu, Japan, in 2003, 2005, and 2008, respectively. He was a research fellow of JSPS in 2006 and 2007. He was an assistant lecturer at Information Systems and Technology Center and Research Center for Advanced Information Science and Technology, University of Aizu in 2008 and 2009, respectively. Since 2010 , he has been an assistant professor at Research Institute of Electrical Communication, Tohoku University, Japan. His research activity is associated with theoretical study and computer simulation of carrier transport in semiconductor nanostructures, including heterostructure two-dimensional electron gas and graphene, for terahertz applications. $\mathrm{He}$ is the author and coauthor of 40 papers in refereed journals. He is a member of the American Physical Society (APS) and IEEE. He received Young Researcher Award at the 1st International Symposium on Terahertz Nanoscience in 2011. 


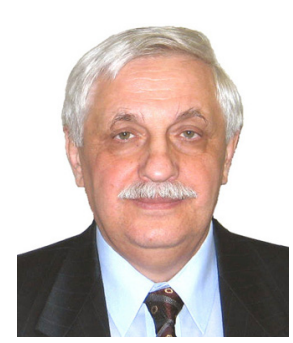

Victor Ryzhii obtained his $\mathrm{PhD}$ and Dr.Sc. (habilitation) degrees from Moscow Institute of Physics and Technology, Russia, in 1970 and 1975, respectively. From 1970 to 1993, he worked in academical and industrial institutions in Russia. In 1993, he joined the University of Aizu, Japan, where he worked as a professor until 2012. Currently, he is a professor emeritus of the University of Aizu and a visiting professor of the Research Institute of Electrical Communication, Tohoku University, Japan. His research activity is associated with physics and computer modeling of low-dimensional semiconductor heterostructures and electronic, optoelectronic, and terahertz devices based on nanostructures, including graphene-based devices. He has about 300 journal publications, numerous conference papers, and 11 patents. He is a fellow of IEEE, APS, and the Institute of Electrical and Electronics Engineers, Japan, a corresponding member of the Russian Academy of Sciences, and a member of the JSAP.

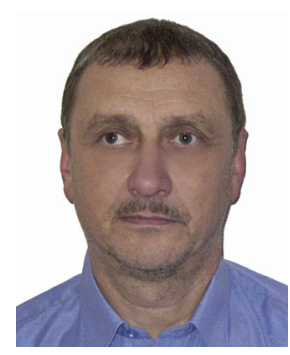

Vyacheslav Popov received the Candidate of Science and Dr.Sc. degrees in radiophysics from Saratov State University, Russia, in 1984 and 1999, respectively. During 1985 to 1990 , he worked as an academic secretary at Regional Council of the Russian Academy of Sciences in Saratov, Russia. Since 1990, he has held a position of head of Advanced Studies Department at Saratov Research Center of the Russian Academy of Sciences. In 1999, he joined Kotelnikov
Institute of Radio Engineering and Electronics of the Russian Academy of Sciences, where he is currently the head of Photonics Laboratory. Since 1999, he is a professor at Physics Department of Saratov State University. He has published more than 100 papers in peer-reviewed journals in the field of theory and modeling of electromagnetic phenomena in microelectronic and nanoelectronic devices.

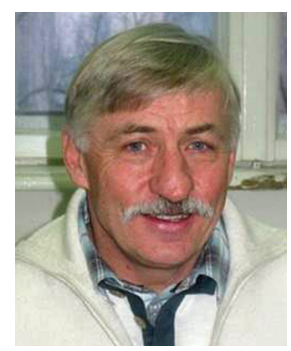

Wojciech Knap obtained his master's degree from the Faculty of Physics, Warsaw University Poland and got a permanent assistant professor position in the Experimental Solid State Physics Department. His PhD concerned the terahertz (far-infrared) properties of narrow gap semiconductors $\mathrm{HgTe}$ and nSb. In 1987, he left to France and worked at University of Montpellier, Grenoble High Magnetic Field Laboratory, Toulouse Pulsed High Magnetic Field Laboratory. In 1992, he obtained a permanent position at French National Center for Scientific Research, Montpellier, where he works now. Between 1999 and 2001, he worked (sabbatical) at USA-Rensselaer Polytechnic Institute. He also obtained a long-term grant from Japan Society of Promotion of Science and spent a year at Tohoku University (2007 to 2008). His main scientific interests are (1) far-infrared (terahertz) properties of semiconductors, (2) quantum phenomena in transport, and (3) terahertz plasma excitations in low-dimensional structures. 